\title{
Malacoplaquia vesical y uracal que simula un adenocarcinoma vesical
}

\section{Malakoplakia of the bladder and urachus simulating bladder adenocarcinoma}

Alan de Jesús Martínez-Salas, Mauricio Cantellano-Orozco, Gabriel Pacheco-Gahbler, Gerardo FernándezNoyola, Carlos Martínez-Arroyo, Gustavo Morales-Montor y colaboradores.

\begin{abstract}
Resumen
ANTECEDENTES: La malacoplaquia es una enfermedad relacionada con la actividad anormal de la función fagocitaria de los macrófagos, que suele afectar la vía urinaria y gastrointestinal, debido a infecciones crónicas (50\%) o inmunosupresión (40\%).

CASO CLínICO: Paciente masculino de 44 años, que acudió al servicio de Urgencias por fiebre $\left(39^{\circ} \mathrm{C}\right)$ de 7 días de evolución, asociada con disuria y dolor abdominal infraumbilical, tipo cólico, intensidad 4/10, con masa periumbilical y leucocitosis. La urotomografía reportó defecto de llenado vesical y comunicación hacia la pared anterior abdominal. El tratamiento consistió en resección transuretral vesical; se resecó el tumor del domo y la pared anterior vesical, con salida de material mucoso. El paciente persistió con fiebre y dolor abdominal posoperatorio, por lo que se decidió efectuar una laparotomía exploradora, en la que se encontró una tumoración abdominal dependiente de la vejiga y el uraco, además de apendicitis reactiva. El reporte histopatológico fue malacoplaquia vesical y uracal.
\end{abstract}

CONCLUSIÓN: Este el primer caso de malacoplaquia uracal con manifestación atípica reportado en el Hospital General Dr. Manuel Gea González.

PALABRAS CLAVE: Malacoplaquia; vejiga; uraco.

\section{Abstract}

BACKGROUND: Malakoplakia is a disease related to the abnormal activity of the phagocytic function of the macrophages that usually affects the urinary and gastrointestinal tracts and is associated with chronic infections (50\%) or immunosuppression $(40 \%)$.

CLINICAL CASE: A 44-year-old man came to the emergency service complaining of fever $\left(39^{\circ} \mathrm{C}\right)$ of 7 -day progression, accompanied by dysuria and colicky, infraumbilical abdominal pain of $4 / 10$ intensity, with a periumbilical mass and leukocytosis. Computed tomography urography identified a bladder storage defect and communication into the anterior abdominal wall. Treatment was transurethral resection of the bladder. The tumor was resected from the bladder dome and the anterior bladder wall, with the release of mucous material. The patient persisted with fever and abdominal pain in the postoperative period. Exploratory laparotomy revealed an abdominal tumor dependent on the bladder and the urachus, as well as reactive appendicitis. The histopathologic report stated malakoplakia of the bladder and urachus.

CONCLUSION: This is the first case of urachal malakoplakia with an atypical presentation reported at the Hospital General Dr. Manuel Gea González.

KEYWORDS: Malakoplakia; Bladder; Urachus
División de Urología, Hospital General Dr. Manuel Gea González (SSA), Ciudad de México.

Recibido: noviembre 2017

Aceptado: mayo 2018

Correspondencia

Alan de Jesús Martínez Salas alanmsalas19@gmail.com

Este artículo debe citarse como Martínez-Salas AJ, Cantellano-Orozco $M$, Pacheco-Gahbler G, FernándezNoyola G, Martínez-Arroyo C, MoralesMontor $\mathrm{G}$ y col. Malacoplaquia vesical y uracal que simula un adenocarcinoma vesical. Rev Mex Urol. 2018 mayojunio;78(3):225-228.

DOI:https://doi.org/10.24245/revmexurol.v78i3.1777 


\section{ANTECEDENTES}

El uraco es un remanente de la extensión embrionaria, localizado entre el alantoides y el seno urogenital; su longitud promedio es de $5 \mathrm{~cm}$ y tiene estructuras fibrosas que lo conectan con la cicatriz umbilical, conocidas como "plexo de Luschka". Durante su trayecto puede ubicarse en el espacio de Retzius, entre la fascia transversalis y el peritoneo parietal. Histológicamente se compone por 3 capas: una interna de epitelio cilíndrico productor de moco, una intermedia con fibras longitudinales musculares y una externa de tejido conectivo. ${ }^{1,2}$ Las alteraciones uracales se subdividen en dos grandes grupos: 1) neoplásicas (suelen afectar a $67 \%$ de los pacientes) y 2) inflamatorias o infecciosas. Entre las enfermedades uracales adquiridas no neoplásicas se encuentran: 1) seno uracal umbilical, 2) divertículo vesicouracal y 3) quiste uracal, que pueden aparecer con diversos procesos inflamatorios o infecciosos. ${ }^{3,4}$

Los síntomas asociados con mayor frecuencia incluyen: disuria, hematuria y, en ocasiones, episodios de retención aguda de orina relacionada con alguna tumoración abdominal suprapúbica o periumbilical palpable, generalmente no dolorosa. El proceso inflamatorio o infeccioso del uraco, además de los síntomas referidos, suele asociarse con datos francos de síndrome de respuesta inflamatoria sistémica y sepsis; incluso en pacientes con ruptura en la cavidad abdominal puede haber signos de irritación peritoneal y abdomen agudo. ${ }^{3,4} \mathrm{El}$ tratamiento inicial consiste en antibiótico de amplio espectro y, posteriormente, procedimiento endoscópico con resección transuretral del uraco; sin embargo, en pacientes con mala reacción al tratamiento se indica resección de persistencia del uraco y cistectomía parcial del domo vesical. La recidiva en pacientes a quienes no se realiza tratamiento quirúrgico o endoscópico se estima en $31 \%{ }^{3}$
La malacoplaquia es una alteración poco estudiada y reportada, solo existen pequeñas series en la bibliografía; por tanto, se desconoce su incidencia y prevalencia. La malacoplaquia se refiere a la actividad deficiente de la función fagocitaria, particularmente de los macrófagos, caracterizada por depósitos de calcio y partículas intracelulares que pueden visualizarse mediante estudio histopatológico, con los Ilamados "cuerpos de Gutmann-Michaelis" e "histiocitos de Von Hasemann". 5-8 La malacoplaquia suele afectar el aparato genitourinario y gastrointestinal, cuya manifestación sugiere una alteración inflamatoria. Casi todos los reportes indican afectación en hombres mayores de 50 años de edad (1.6:1), comúnmente asociada con infecciones crónicas por Escherichia coli $(50 \%)$ e inmunosupresión $(40 \%) .{ }^{5}$ Hasta la fecha no existen guías o recomendaciones terapéuticas para esta enfermedad; sin embargo, se han prescrito antibióticos con acción bactericida contra organismos intracelulares, particularmente quinolonas y trimetoprima-sulfametoxazol, y en pacientes con mala reacción al tratamiento se ha planteado la necesidad de endoscopia, incluso procedimientos quirúrgicos. ${ }^{5}$

A continuación se describe un caso poco frecuente de malacoplaquia uracal y vesical, similar a una tumoración abdominal, y se revisa la bibliografía relacionada con esta alteración.

\section{CASO CLÍNICO}

Paciente masculino de 44 años de edad, que acudió al servicio de Urgencias por fiebre de $39^{\circ} \mathrm{C}$, de siete días de evolución, asociada con disuria y dolor abdominal infraumbilical tipo cólico (intensidad 4/10). A la exploración física se palpó una tumoración infraumbilical, con percepción de dolor moderado, ahulada, móvil, no fluctuante o crepitante. Los estudios de laboratorio reportaron leucocitocis de $27,000 / \mu \mathrm{L}$ y el examen general de orina objetivó leucocituria. leucocituria como único hallazgo patológico; el urocultivo no evidenció crecimiento bacteriano. 
La urotomografía evidenció un defecto de llenado vesical, dependiente del domo, y comunicación hacia la pared anterior abdominal, donde se visualizó una tumoración abdominal adherida al domo vesical (Figuras 1 y 2). Se llevó a cabo cistoscopia y resección transuretral vesical (Figura 3), de la que se extirpó una tumoración dependiente del domo y la pared anterior vesical con salida de material mucoso.

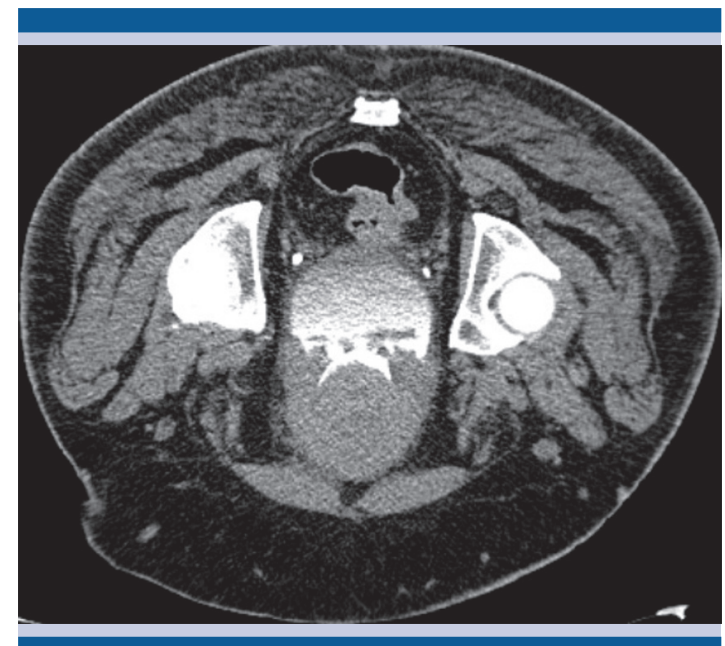

Figura 1. Tumoración dependiente del domo vesical con defecto de llenado en fase de eliminación.

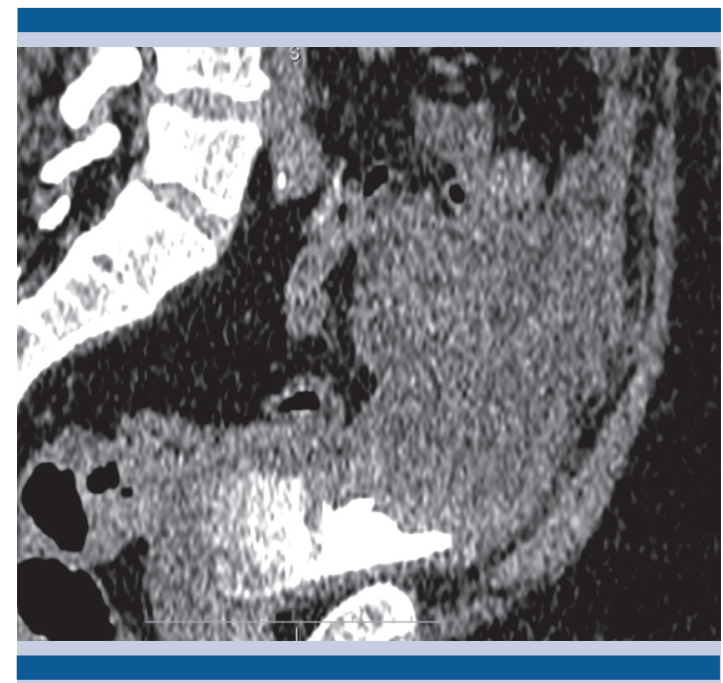

Figura 2. Tumoración abdominal conectada con el domo vesical, con defecto de llenado.

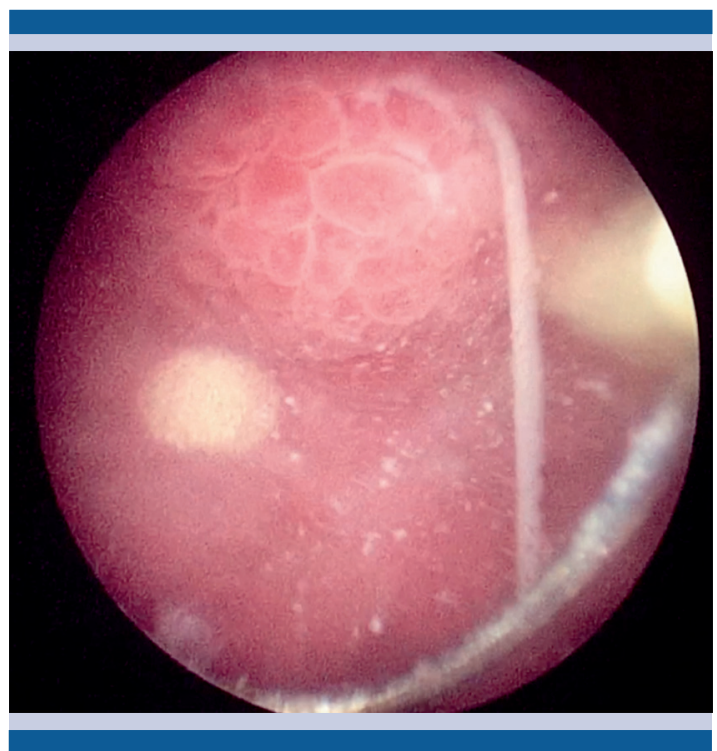

Figura 3. Cistoscopia que evidencia la lesión en el domo vesical con salida de contenido mucoso.

Durante el posoperatorio persistió con fiebre y dolor abdominal, además de leucocitosis y datos de irritación peritoneal. En la laparotomía exploradora se identificó una tumoración abdominal dependiente de la vejiga y el uraco, con apendicitis reactiva, que fue resecada en su totalidad con cistectomía parcial del domo vesical. El reporte histopatológico fue malacoplaquia vesical y uracal (Figura 4).

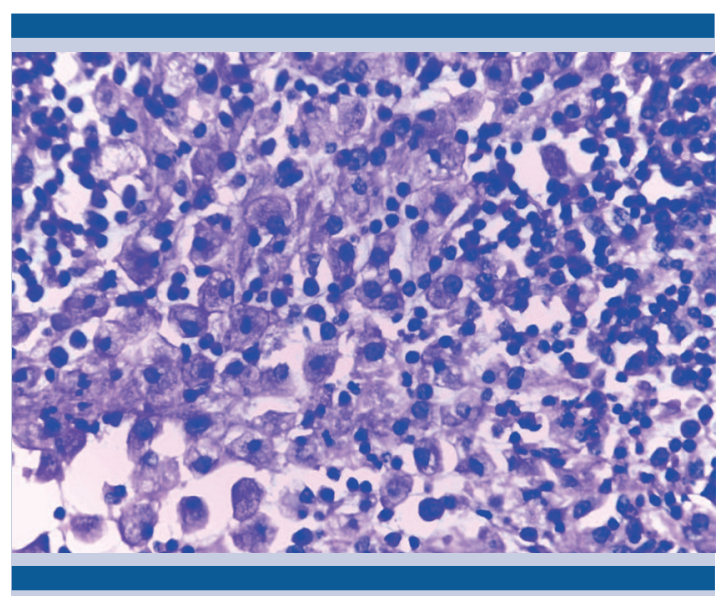

Figura 4. Abundantes macrófagos e histiocitos con abundante contenido de calcio y depósitos intracelulares compatibles con malacoplaquia. 


\section{DISCUSIÓN}

El caso aquí expuesto refiere una variante excepcional de malacoplaquia uracal y vesical, que simuló una tumoración abdominal. Las principales alteraciones malignas del uraco incluyen al adenocarcinoma y los abscesos. En nuestro caso, según las características del tumor descritas, el diagnóstico diferencial suponía procesos infecciosos dependientes de la vejiga o del uraco. El tratamiento inicial fue adecuado y coincidió con lo descrito en la bibliografía (prescripción de antibióticos y cistoscopia). Algunos autores indican antibióticos de amplio espectro y tratamiento endoscópico; sin embargo, el paciente de este estudio tuvo deterioro del estado general y evolución de los síntomas, por lo que se decidió efectuar la resección de la tumoración. ${ }^{5-8}$ El hallazgo histopatológico fue compatible con malacoplaquia, una enfermedad poco estudiada y reportada en la bibliografía; por tanto, representa un reto diagnóstico multidisciplinario, debido a su manifestación clínica, hallazgos fisiopatológicos e histológicos.

\section{CONCLUSIÓN}

Este es el primer reporte de malacoplaquia uracal y vesical, con simulación de tumoración abdominal, en el Hospital General Dr. Manuel Gea González. Se requieren estudios adicionales para conocer y estudiar esta enfermedad poco frecuente y considerarla en el diagnóstico diferencial de las alteraciones dependientes del uraco.

\section{Colaboradores}

Aldo Jiménez-García, Sebastian Jesús MuruatoAraiza e Irving Reyna-Blanco.

\section{REFERENCIAS}

1. Sterling JA, Goldsmith R. Lesions of urachus which appear in the adult. Ann Surg 1953;137(1):120-8.

2. Blichert-Toft M, Nielsen OV. Diseases of urachus simulating intraabdominal disorders. Am J Surg 1971;122(1):123-8.

3. Mylonas KS, O Malley P, Ziogas IA, El-Kabab L, Nasioudis D. Malignant urachal neoplasms: a population-based study and systematic review of literature. Urol Oncol 2017;35(1):33.e11-33.e19

4. Tazi F, Ahsaini M, Khalouk A, Mellas S, et al. Abscess of urachal remnant presenting with acute abdomen: case series. J Med Case Rep 2012;30(6):226.

5. Tian J, Ma JH, Li CL, Xiao ZD. Urachal mass in adults: clinical analysis of 33 cases. Zhonghua Yi Xue Za Zhi 2008;88(12):820-2.

6. Dong H, Dawes S, Philip J, Chaudhri S, Subramonian K. Malakoplakia of urogenital tract. Urol Case Rep 2015;3(1):6-8.

7. Pakalapati S, Parachuri S, Kakarla VR, Byrappa MD. Isolated urachal malakoplakia mimicking malignancy. Urol Ann 2017;9(1):92-95.

8. Sarkis P, Nawfal G, Mouaccadieh L, Daou I, Zanati M. Malakoplakie rénale bilaterales et vésicale: à propos d'un cas en revue de la littérature. Prog Urol 2012;22(15):970-3 